\title{
Research on Image Denoising with an Improved Wavelet Threshold Algorithm
}

\author{
Wang Qian \\ Zibo Vocational Institute, Zibo, China, 255314 \\ $55976631 @ q q . c o m$
}

\begin{abstract}
Wavelet denoising is a commonly used method in the field of image denoising algorithms, based on the analysis of the characteristics of estimated wavelet coefficients in the wavelet threshold denoising and the traditional soft threshold and hard threshold method, in the light of the discontinuity of hard threshold and fixed deviation of soft threshold, and non-differentiable of compromise algorithm, a wavelet threshold denoising algorithm is put forward which is continuous based on the wavelet coefficients and differentiable and variable threshold value deviation, the experimental results show that this method has better denoising effect and has good practical value.
\end{abstract}

Keywords: Image denoising; Wavelet algorithm; Threshold; Continuous differentiable

\section{Introduction}

In the present era of technology high speed development, the image information in people's work, study and play an increasingly important role in the life. But actually obtain the image in the collection, transmission, receiving and processing of the inevitable exist in the process of the external interference and internal interference, such as photoelectric conversion sensor sensitivity is not uniform, the quantization noise of digital process, in the process of transmission error, etc., there will be a certain amount of noise. How to remove the noise, we get the original image is an important issue in image processing.

People according to the characteristics of the actual images, the statistical characteristic of noise and spectrum distribution characteristics, there are many methods of image denoising is studied in this paper. Many ways, the most direct approach is the low pass filter method, because according to noise belongs to the high frequency component, most of the signal belongs to the low frequency component. So many of the methods were adopted or low pass filtering method is of low pass filtering method to a certain degree of improvement. However, a low-pass filter to eliminate the noise at the same time, also will be a useful part in eliminating signal at high frequency. Therefore, how to get a better balance to remove the noise and retain the high frequency information, $\sim$ straight is the difficulty and hotspot in the research of the scientist.

With a "mathematical microscope" of wavelet because of its time-frequency local features and advantages of multi-resolution analysis, make its constantly in all fields reveal hilf, radius and the growing expansion. So far, its application has many areas around the image processing aspects: image noise reduction, image enhancement, image segmentation, digital watermarking, image reconstruction and image compression coding, etc.

Image denoising problems has been a widely discussed problem in signal processing, image will be different level noise pollution due to reality, in order to improve the SNR of image, highlight the desired features of images, in order to a higher level of processing, the image noise reduction processing is necessary and inevitable. Wavelet analysis of the 
characteristic of the time window and frequency window can change features with the features of multi-resolution analysis can effectively separate signal and noise, is nearly 10 years developed a kind of image denoising methods. Wavelet noise reduction method of success to the low quantity, wavelet sparse distribution of wavelet coefficient reduces the image information quantity; Multi-resolution analysis, can set a different threshold for different scale, can very good deal with discontinuous points and singular points, etc.; To relevance, wavelet transform to signal correlation processing, such as satisfy gaussian noise in wavelet domain, convenient to remove noise; Choose base flexibility, flexible and the generating function of the selection of wavelet transform, the different situation of different processing.

Image data tend to have a variety of information redundancy, such as redundant quantity and visual redundancy, structural redundancy, etc. If the data quantity is large, or require transmission speed, image compression has played a vital role. The purpose of digital image compression is to minimize the amount of data to express the image, but also guarantee the image quality. In the case of high compression ratio, compression image would inevitably lose detail information, different compression technology can produce different types of coding distortion. Wavelet transform has the multi-resolution analysis characteristic of natural and efficient quantitative strategies, such as wavelet zerotree quantization [1]; Can better adapt to the time domain of wavelet coefficient of locality; These features make the wavelet transform has been widely applied in image compression. Applications, the wavelet transform coding at low bit rates, quality of compressed image is superior to the traditional DCT (discrete cosine transform) encoding.

Image data in the acquisition, distribution, transmission and receiving, output, copying process there will be noise, lower the quality of the image. Sometimes to compress image processing, image compression, also will reduce the quality of the image. At this time all need to enhance the image, the image emerged: interested in part of traditional image enhancement algorithms, such as high-pass filtering, histogram mean quantization method in improving image contrast and enhancing of the detail of the image at the same time also magnifies the noise. Image enhancement based on wavelet analysis, the feature of wavelet multi-resolution analysis, in the process, the high frequency adopting gradually the temporal and spatial step length, can enhance the high frequency component or weaken the low frequency components [2].

Digital watermarking is in recent years, along with the rising popularity of the Internet, multimedia information exchange reached unprecedented depth and breadth, but works infringement is easy with tamper with the more convenient also, under this background, the technology of digital products copyright protection [3]. This technology is currently is a hot spot in scientific research. Digital watermarking is mainly to prove that, perceptual, robustness and other features. The research direction of digital watermark embedding and extraction mainly is the spatial domain watermark, transform domain algorithm, compressed domain algorithm, NEC (NEC laboratories Cox and others) algorithm. Because the wavelet analysis of the wavelet base selection and time-frequency locality, academics have applied wavelet analysis in sub image watermarking technology, and got good results.

Methods such as simple image fusion algorithm has weakened the details of image, reduces the contrast of the image, to a certain extent, made in the image edge blur, in most applications fusion effect is satisfactory. Based on wavelet multi-scale feature of image fusion technology research and application is very popular with people, and the three representative fusion algorithm of wavelet transform, the standard wavelet fusion algorithm, based on the sampling of discrete wavelet transform fusion algorithm ${ }^{[4]}$, based on dual tree complex wavelet transform fusion algorithm.

Wavelet analysis is nearly 15 years developed a kind of new method of time-frequency analysis [5]. Wavelet analysis by I.D aubechies, al-qeada rossman and Y.M eyer et groundwork has been developed rapidly and the $70 \mathrm{~s}$, al-qeada rossman and Y.M eyer and 
others when doing data processing is proposed for the first time the concept of "wavelet", then constructs the $\mathrm{L}^{2}(\mathrm{R})$ specification [6] is intersection, since then got the widespread approval of wavelet. Later, scientists have proposed the wavelet multi-resolution analysis and orthogonal wavelet function, wavelet packet and spline wavelet concept that wavelet analysis get step into $\sim$ the popularization and improvement. Since then, along with scientists and put forward a compactly supported biorthogonal wavelet, wavelet cycle, interval wavelet, wavelet and so on a variety of wavelet basis function, people more and more research on the wavelet, wavelet analysis theory is becoming more and more mature, the application of wavelet analysis is becoming more and more widely.

\section{Related Work}

Introduce wavelet, we can't pass Fourier transform, Fourier transform is more familiar to everyone, Fourier transform is a kind of global transformation, can signal in time domain and frequency domain respectively to observe, but cannot be analyzed signal time-frequency local features, performance in know signal don't know when the time domain waveform of the signal of any frequency domain information, the same frequency characteristic of signals, don't know any time signal characteristics. Such Fourier transform in the signal processing is a basic contradiction, namely the time domain and frequency domain localization of contradictions. However, in practical applications, the signal only from the view of time domain or frequency domain analysis is not enough, especially for non-stationary signal processing, such as sudden transformation, signal instantaneous transformation point, signal the beginning of the end, and so on and so forth, determined by the Fourier transform of time domain and frequency domain are not enough. In order to solve this problem, people put forward many methods, one of the most effective for Fourier transform on the basis of the proposed method of short time Fourier transform (STFT). In simple terms is a short time Fourier transform (STFT) : assume that based on the analysis of non-stationary signal window function $\mathrm{g}(\mathrm{t})$ in a short time interval is smooth, mobile analysis window function $g(t)$, the $f(t) g(t-s)$ in different within finite time width is stationary signal, and the power spectrum of different time is calculated. But using the short time Fourier transform (STFT) fixed time window function, although there has been a great progress, but still defects and deficiencies in signal analysis [7].

Then wavelet transform theory is put forward, it is kind of signal time scale analysis method, the performance in time domain and frequency domain can be the ability of denoting local signal characteristics. The resolution analysis characteristics, realizing the adjustment scale changes from big to small, observe the target at each scale.

Wavelet transform has four important features: the time-frequency localization characteristics, multi-resolution characteristics, the characteristics and the diversity of the wavelet base selection. According to the different characteristics and small potter fusion, has formed three major kinds of noise reduction method based on wavelet analysis: threshold de-noising method (threshold shrinkage method), correlation noise reduction method, the modulus maxima de-noising method.

\section{(1) Threshold denoising method}

In early 90, after the Mallat modulus maxima de-noising method is proposed, Donoho et al proposed threshold de-noising method based on wavelet transform. After a lot of people to continuously improve. This article is in front of a lot of improvement method, on the basis of a new improved method is proposed. Until now, the threshold denoising method has become the most widely used is the most effective method for image denoising. The process for the noise signal after wavelet decomposition, select the appropriate threshold, retains the wavelet coefficient of signal, and make the most noise 
wavelet coefficients to zero. The classical threshold de-noising method (soft threshold method) can be divided into three steps [8]:

1. Signal decomposition. Choose a wavelet generating function and to determine the layer number of wavelet decomposition of $\mathrm{N}$, and then the signal wavelet decomposition for $\mathrm{N}$ layer;

2. To deal with wavelet decomposition coefficient. High frequency coefficient of shrinkage of the $1 \sim \mathrm{N}$ layer processing, to keep low frequency coefficient, after processing of wavelet coefficient. This step is the key problem is to choose the size of the threshold and threshold shrinkage method.

3. Use after processing the wavelet coefficients of signal reconstruction, after de-noising signals.

4. Proven by practice, threshold de-noising method is suitable for the high signal noise ratio (SNR).

\section{(2) Based on wavelet transform correlation of noise reduction}

Wavelet transform image correlation is mainly divided into three categories: the correlation of scale, dimension asked correlation and at the same time, considering the correlation between scale and scale. Scale the relevance of performance in the same subband of wavelet coefficient has gathered properties; The correlation between scale of different scales in the same direction there is a correlation between wavelet coefficients: ask at the same time considering the scale and scale coefficient of correlation can be through the establishment of contains coefficient, coefficient and adjacent coefficients of function to measure.

Of the multi-dimension relevance was applied more widely, its based on wavelet transform coefficients of signal and noise in different scales of features, according to the relevant characteristics of adjacent scale wavelet coefficient, specify different criteria, according to the criterion of wavelet coefficients for processing. Signal wavelet transform coefficient on different scales with different propagation characteristics, for the signal, the wavelet coefficient has a strong correlation, and for the noise, the correlation of wavelet coefficients is very weak.

Related denoising by comparing is the most important link in the normalized correlation coefficient and the size of the absolute value of wavelet coefficient to extract the signal of edge information. Because the normalized correlation coefficient and wavelet coefficients with the same energy, so after compare the two remaining is noise, after many iterations, the energy of the wavelet coefficients below a certain threshold, then think signal has been completely extracted.

Correlation denoising specific steps [9]:

1. The wavelet decomposition function called for signal decomposition, get approximation coefficients and detail coefficients b;

2. The initialize all the correlation coefficient and regularization correlation coefficient;

3. The estimated noise threshold, and to extract the edge information of each layer;

4. Call the wavelet reconstruction function to reconstruct the signal, after denoising signal.

Relevant denoising method is not applicable to high signal noise ratio (SNR), use this kind of situation, there will be not indeed improve signal-to-noise ratio.

(3) The modulus maxima de-noising method

Modulus maxima denoising method[10] by S.M initially allat is put forward, applied the singularity of the signal and noise characteristics, by selecting the singularity of the wavelet transform modulus maxima, according to the wavelet coefficients of signal and 
noise Lipschitz index, remove the noise generated by the wavelet transform maximum, to reconstruct the useful signal.

In this algorithm, the wavelet transform coefficients in different scales have different pattern: a $>0$, the wavelet transform coefficients increase with the increase of scale; a $<0$, the wavelet transform coefficient decreased with the increase of the scale. Commonly used signal of Lipschitz index greater than zero, and white noise Lipschitz index a $=a$ 1/2 $-s,(s)>0$, so according to the different scales of the wavelet transform modulus maxima of change law of signal and noise separation, realize the denoising.

\section{The Wavelet Threshold Algorithm Improved Model}

Define $\mathrm{f}(\mathrm{x})$ as the measured signal,

$$
f(x)=s(x)+n(x)
$$

In which, $\mathrm{f}(\mathrm{x})$ is the signal with noise, $\mathrm{s}(\mathrm{x})$ is the original signal, $\mathrm{n}(\mathrm{x})$ is the Gauss noise.

$f(x)$ is wavelet decomposed in each scale, on the scale of the wavelet coefficients are processed by threshold functions, the final will be processing the wavelet coefficients obtained using the inverse wavelet transform reconstruction, recovering the useful signal. Selection of threshold function is different, is embodied in different processing of wavelet coefficient of strategy, but the basic idea is to remove small coefficient, the coefficient of the proper "shrinkage" or retained.

As is known to all, the wavelet coefficients of noise is inversely proportional to the decomposition scale, namely, with the increase of wavelet decomposition scale threshold should be gradually reduced. Here we adopts the definition of Donoho [11]

$$
\lambda=\delta \sqrt{2 \log (n) / n}
$$

In which $\sigma$ (noise standard variance) is

$$
\sigma=M A D / 0.6745
$$

MAD is detail wavelet coefficients of the median, $\mathrm{n}$ is the product of signal aspect of pixels.

The current way of threshold value has four kinds:

(1) The rigrsure rule: on the basis of the principle of Stein's unbiased likelihood estimation for adaptive worshiping value choice. Its processing results tend to be "reserved" wavelet coefficients, and obtained the optimal threshold value tends to be the ideal threshold.

(2) The sqtwolog rule: in the form of a fixed threshold of $\sqrt{2 \log (\operatorname{length}(x))}$

(3) The heursure rule: the first two threshold of comprehensive, choose the optimal predictor variable threshold.

(4) The mimimaxi rule: fixed threshold selection form, create a minimum mean square deviation of extreme value. It generally rely on the original signal and showed no expression, the tendency of wavelet coefficients, exists in using this threshold denoising effect is often not satisfactory.

Threshold value of the most common processing method is Donoho hard threshold and soft threshold method and method of compromise [12].

$$
\begin{gathered}
w^{\prime}=\left\{\begin{array}{l}
\operatorname{sgn}(w)(|w|-\lambda),|w| \geq \lambda \\
0,|w|<\lambda
\end{array}\right. \\
w^{\prime}=\left\{\begin{array}{l}
w,|w| \geq \lambda \\
0,|w|<\lambda
\end{array}\right.
\end{gathered}
$$




$$
w^{\prime}=\left\{\begin{array}{l}
\operatorname{sgn}(w)(|w|-a \lambda),|w| \geq \lambda \\
0,|w|<\lambda
\end{array}\right.
$$

In which, $w^{\prime}$ is threshold, $w$ is wavelet coefficients, $0<\mathrm{a}<1$, use this method to get the wavelet coefficients in the adjustable range deviation is greater than $\lambda$, it is called compromise with the deviation is between 0 and $\lambda$.

Soft and hard threshold method exist some defects in practice: soft threshold continuity on the whole, but after denoising, some of the signal singularity submerged by noise easily, and the image will appear false visual distortion phenomenon such as gibbs effect, soft threshold function is greater than the setting threshold wavelet coefficients for constant bias compression, a direct impact on the reconstructed signal and real signal approximation degree. Hard threshold function is discontinuous at $\lambda$ and $-\lambda$, received signal will generate additional oscillation, and the greater than the threshold of wavelet coefficients do not do processing, which may have residual noise signal. Compromise method in $0<a<1$, enlarged the range of deviation, experiments show that better denoising effect, but only take the boundary function that can guide $\mathrm{a}=1$. In fact, math derivation operation, the threshold function is frequently the above three methods have shortcomings.

Because of the soft threshold method after processing of wavelet coefficient is continuous, on this basis, construct new threshold method, satisfy threshold function is continuous and differentiable characteristic, coefficient equations satisfy the following formula [13],

$$
w^{\prime}=\left\{\begin{array}{l}
0,|w| \leq m \\
\operatorname{sgn}(w) C(|w|), m<|w|<n \\
\operatorname{sgn}(w)(|w|-a \lambda),|w| \geq n
\end{array}\right.
$$

In which, $C(|w|)$ is quadratic polynomial, $A x^{2}+B x+C$, and satisfy

$C(|m|)=0, C(|m|)^{\prime}=0, C(|n|)^{\prime}=1, m<n$, so we can conclude that

$$
C(x)=\frac{1}{2(n-m)} x^{2}-\frac{m}{n-m} x+\frac{m^{2}}{2(n-m)}
$$

Analysis to get $C(|n|)=(n-m) / 2=n-m \lambda, m+n=2 a \lambda$ means that there are two variables amount $\mathrm{m}, \mathrm{n}$ and $\mathrm{a}$, when $m=\lambda, n=(2 a-1) \lambda$, a can be taken as a appropriate value, when a is tend infinitely to 0.5 , the function is turned into soft threshold function. Combining threshold estimation function of $\mathrm{m}$ and a selection of appropriate values, the highest SNR. Improved threshold method to achieve the threshold value function of continuity, overcomes and discontinuity point of problem, can satisfy the practical application of threshold function derivation operation, to greater than the coefficient of processing, the use of the coefficient of the optional compromise threshold method.

\section{Experiment and Analysis}

In order to do data contrast, we have to manually add Lena image noise. Add noise to zero mean gaussian white noise and random noise. To deal with the noise in noise image, we will be calculated after denoising image and PSNR of the image before adding noise, as image of the judging criteria, which are defined as follows ${ }^{[14]}$ :

$$
M=\frac{1}{\mathrm{~m} \times \mathrm{n}} \sum_{i=1}^{m} \sum_{j=1}^{n}\left(\mathrm{f}(\mathrm{i}, \mathrm{j})-N(\mathrm{i}, \mathrm{j})-X^{\prime}(\mathrm{i}, \mathrm{j})\right)^{2}
$$




$$
P N S R=-10 \lg \frac{M}{255^{2}}
$$

For variance among them, as the peak signal-to-noise ratio, PSNR is the most common and most widely used the objective measurement method of the evaluation of image quality, but many experimental results show that PSNR unable to score and the visual quality of the human eye to see completely consistent, likely PSNR high looks worse than PSNR lower instead. This is because the human eye vision for the sensitivity of the error is not absolute, the perceptual result will be influenced by many factors, resulting in changes (for example: the human eye to empty asked low frequency contrast sensitivity difference is higher, the sensitivity of the human eye to brightness contrast difference is relatively high colority, perception of the human eye to a regional results will be affected by the adjacent area around). Therefore, PSNR can only as a measure of image quality standard, the stand or fall of image is correlated with human's subjective feeling.

Table 1 and table 2 respectively show the result of polluted Lena image after adding two kinds of noise, with soft and hard threshold and compromise method and improved method proposed in this paper it is concluded that the SNR and PNSR contrast.

Table 1. The Filtered Gaussian Noise Image SNR and RMSE Contrast

\begin{tabular}{|c|c|c|c|c|c|}
\hline & $\begin{array}{c}\text { Source } \\
\text { image }\end{array}$ & $\begin{array}{c}\text { Hard } \\
\text { threshold }\end{array}$ & $\begin{array}{c}\text { Soft } \\
\text { threshold }\end{array}$ & Split method & New method \\
\hline SNR & 18.599 & 19.439 & 18.478 & 19.388 & 20.377 \\
\hline PSNR & 0.382 & 13.392 & 14.465 & 13.283 & 12.357 \\
\hline
\end{tabular}

Table 2. Random Noise Filtered SNR and RMSE Contrast

\begin{tabular}{|l|l|l|l|l|l|}
\hline & \multicolumn{1}{|c|}{$\begin{array}{c}\text { Source } \\
\text { image }\end{array}$} & $\begin{array}{c}\text { Hard } \\
\text { threshold }\end{array}$ & $\begin{array}{c}\text { Soft } \\
\text { threshold }\end{array}$ & Split method & New method \\
\hline SNR & 20.459 & 21.483 & 20.392 & 21.658 & 22.377 \\
\hline PSNR & 0.483 & 11.468 & 13.457 & 11.468 & 10.985 \\
\hline
\end{tabular}

Can be seen from the table above, the paper puts forward the improved method is better than the soft hard threshold and compromise method denoising effect.

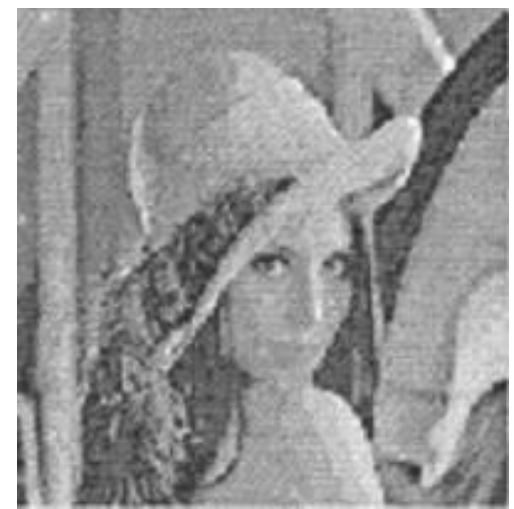

1 Source image

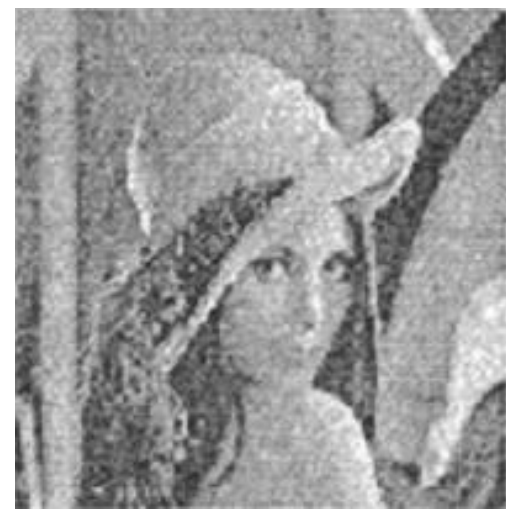

2 Add Gauss noise 


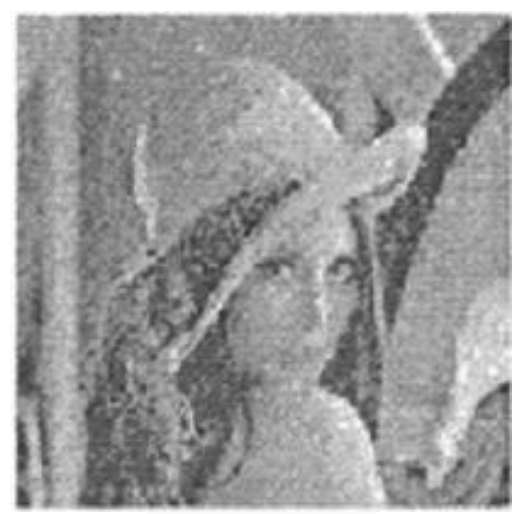

3 Hard threshold

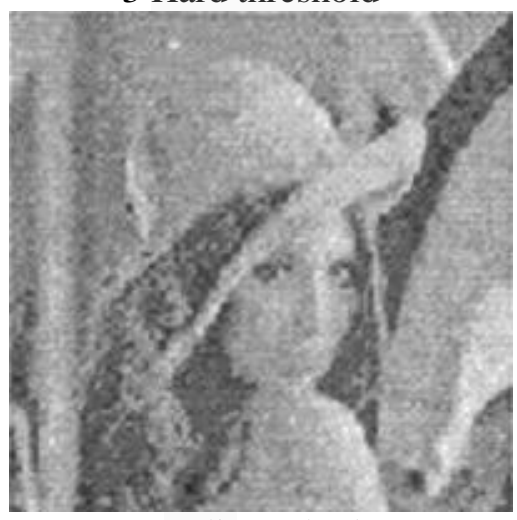

5 Split method

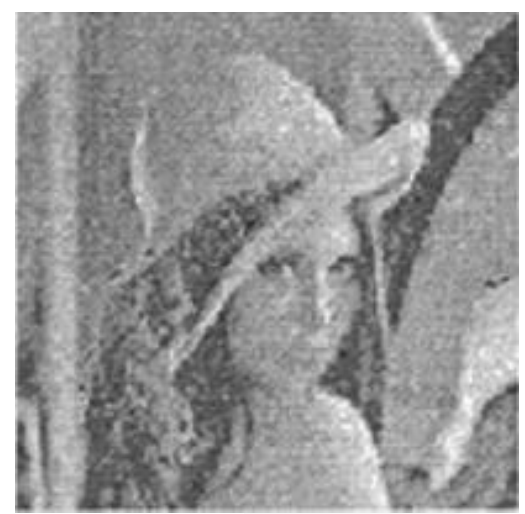

4 Soft threshold

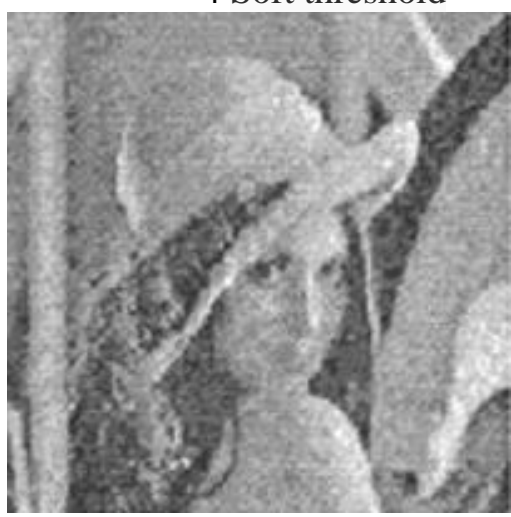

6 New method

Figure 1. Image Denoising Effect Contrast Figure

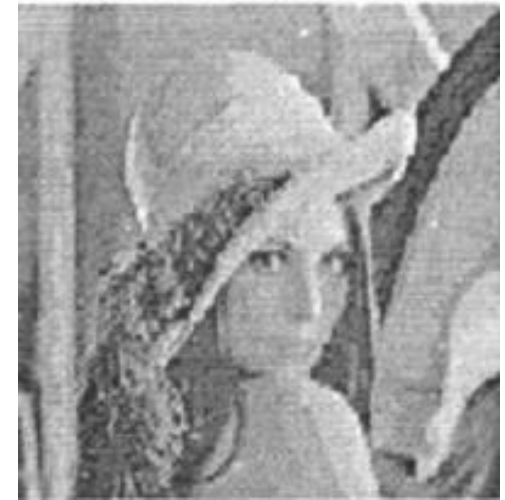

1 Source image

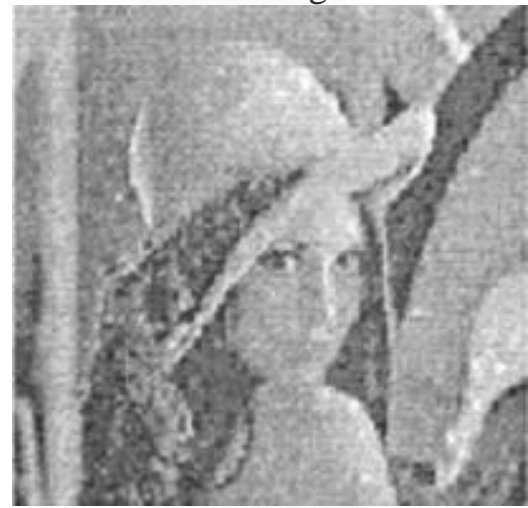

3 Hard threshold

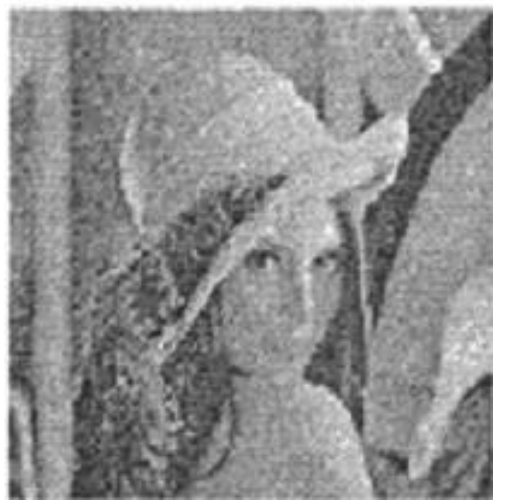

2 Add random noise

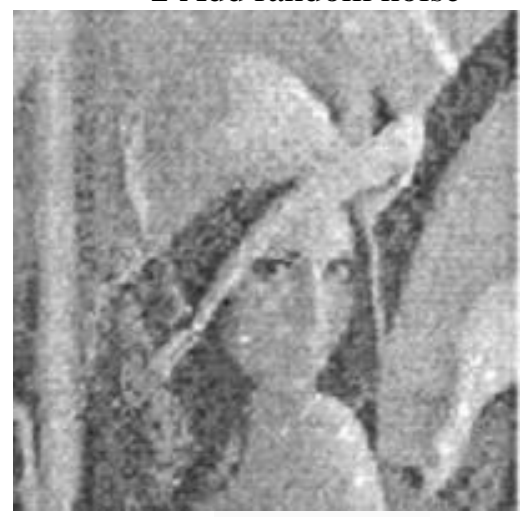

4 Soft threshold 


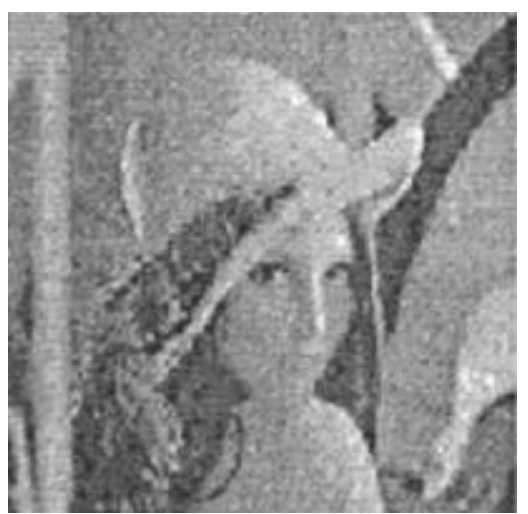

5 Split method

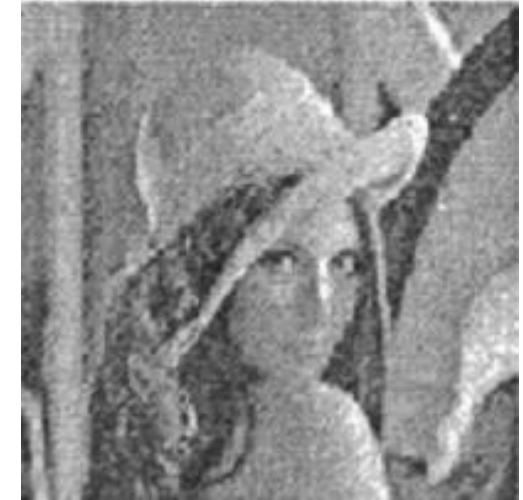

6 New method

Figure 2. Image Denoising Effect Contrast Figure

The algorithm based on the analysis of the traditional soft in value and hard min value method, on the basis of based on the discrete characteristics of fujian value hard and soft min value of a fixed deviation, and compromise algorithm not conductivity is put forward based on the wavelet coefficients of continuous and differentiable and deviation variable threshold wavelet threshold de-noising method. The algorithm fully advantage of the characteristics of the wavelet transform, analyze the characteristics of the wavelet coefficients are estimated, to fujian and wavelet de-noising algorithm is improved. The experimental results show that the method is simple, small amount of calculation and low time complexity, the denoising effect is good.

\section{Conclusion}

Based on wavelet denoising is commonly used in image denoising method, through the analysis in the wavelet threshold denoising to estimate the characteristics of wavelet coefficients, the traditional soft threshold and hard threshold method, on the basis of based on the discrete characteristics of hard threshold and soft threshold fixed deviation, and compromise algorithm not conductivity is put forward based on the wavelet coefficients of continuous and differentiable and variable threshold value deviation of worshiping wavelet denoising method, the experimental results show that the method have good denoising effect, should have good practical value.

\section{References}

[1] J L. Starck, E J. Candès and D L. Donoho, "The curvelet transform for image denoising [J]", Image Processing, IEEE Transactions on, vol. 11, no. 6, (2002), pp. 670-684.

[2] H. Li, B S. Manjunath and S K. Mitra, "Multisensor image fusion using the wavelet transform [J]", Graphical models and image processing, vol. 57, no. 3, (2005), pp. 235-245.

[3] A S. Lewis and G. Knowles, "Image compression using the 2-D wavelet transform [J]", Image Processing, IEEE Transactions on, vol. 1, no. 2, (2006), pp. 244-250.

[4] S G. Chang, B. Yu and M. Vetterli, "Adaptive wavelet thresholding for image denoising and compression [J]", Image Processing, IEEE Transactions on, vol. 9, no. 9, (2000), pp. 1532-1546.

[5] A. Buades, B. Coll and J M. Morel, "A review of image denoising algorithms, with a new one [J]", Multiscale Modeling \& Simulation, vol. 4, no. 2, (2005), pp. 490-530.

[6] Y H. Lee and S A. Kassam, "Generalized median filtering and related nonlinear filtering techniques [J]", Acoustics, Speech and Signal Processing, IEEE Transactions on, vol. 33, no. 3, (2006), pp. 672-683.

[7] O. Rockinger, "Image sequence fusion using a shift-invariant wavelet transform [C]", Image Processing, 2005, Proceedings, International Conference on. IEEE, vol. 3, (2005), pp. 288-291.

[8] J. Portilla, V. Strela and M J. Wainwright, "Image denoising using scale mixtures of Gausss in the wavelet domain [J]”, Image Processing, IEEE Transactions on, vol. 12, no. 11, (2003), pp. 1338-1351.

[9] M. Elad and M. Aharon, "Image denoising via sparse and redundant representations over learned dictionaries [J]", Image Processing, IEEE Transactions on, vol. 15, no. 12, (2006), pp. 3736-3745. 
[10] T. Huang, G. Yang and G. Tang, "A fast two-dimensional median filtering algorithm [J]", Acoustics, Speech and Signal Processing, IEEE Transactions on, vol. 27, no. 1, (2005), pp. 13-18.

[11] A C. Bovik, T S. Huang and D C. Munson Jr., "A generalization of median filtering using linear combinations of order statistics [J]", Acoustics, Speech and Signal Processing, IEEE Transactions on, vol. 31, no. 6, (2006), pp. 1342-1350.

[12] M. Antonini, M. Barlaud and P. Mathieu, "Image coding using wavelet transform [J]", Image Processing, IEEE Transactions on, vol. 1, no. 2, (2007), pp. 205-220.

[13] K. Dabov, A. Foi and V. Katkovnik, "Image denoising by sparse 3-D transform-domain collaborative filtering [J]”, Image Processing, IEEE Transactions on, vol. 16, no. 8, (2007), pp. 2080-2095.

[14] A. Grinsted, J C. Moore and S. Jevrejeva, "Application of the cross wavelet transform and wavelet coherence to geophysical time series [J]", Nonlinear processes in geophysics, vol. 11, no. 5/6, (2004), pp. 561-566.

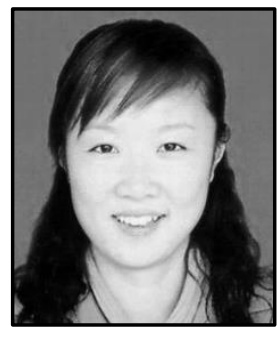

Wang Qian, she received the B.S. degree in Computer science and technology from Huanggang Normal University and the MPA degree of Software engineering from University of Electronic Science and technology of China in 2003 and 2010 respectively. She is lecturer at Zibo vocational institute where she is backbone teacher of software department. She is currently researching on algorithm design, Information and software project development, Network engineering, etc. 